Research Article

\title{
Design of Standoff Cooperative Target-Tracking Guidance Laws for Autonomous Unmanned Aerial Vehicles
}

\author{
Zhen Li $\mathbb{D}^{\text {, }}$, Xin Chen, and Zhenhua Zhao \\ College of Automation Engineering, Nanjing University of Aeronautics and Astronautics, Nanjing 211106, China \\ Correspondence should be addressed to Zhen Li; lizhenbx1503025@nuaa.edu.cn
}

Received 4 December 2020; Revised 20 March 2021; Accepted 17 April 2021; Published 3 May 2021

Academic Editor: Haibo Du

Copyright ( ) 2021 Zhen Li et al. This is an open access article distributed under the Creative Commons Attribution License, which permits unrestricted use, distribution, and reproduction in any medium, provided the original work is properly cited.

\begin{abstract}
This paper investigates two guidance laws of standoff cooperative tracking static and moving of multiple autonomous unmanned aerial vehicles for targets from the perspective of the control system design. In the scheme of the proposed guidance laws, one vehicle is chosen as leader and others as followers. The leader only needs the measurement of the target, and the followers only measure the leader and its neighbors in the communication topology network. By using the proposed guidance laws, it is guaranteed that all vehicles can track a static or moving target with an evenly spaced formation of circle. Considering the coupling of tracking and cooperation, the stability analysis is performed by constructing two relatively independent subsystems based on Lyapunov theory, and the corresponding rigorous proofs of stability are given. By comparing with the Lyapunov vector field guidance law, the simulation results verify the effectiveness and superiority of the proposed guidance laws.
\end{abstract}

\section{Introduction}

In the past few decades, due to the rapid development of science and technology, the use of unmanned aerial vehicles (UAVs) is from surveillance and reconnaissance to rescue and communication relay [1-3], and its application fields have been greatly expanded. At present, one of the most important applications of UAVs is the use of tracking ground targets; through the UAV guidance system, the target can be always in the field of view, so as to achieve realtime tracking purpose of ground targets [4-6]. Compared with single UAV, multi-UAV cooperative tracking can increase the coverage of the sensor on the ground target and reduce the target state estimation error, and it has a wider range of application prospects $[7,8]$.

Unlike rotary-wing UAV, fixed-wing UAV must maintain a minimum velocity to produce enough lift. A typical way to accomplish a tracking mission is standoff tracking which means to monitor the ground target by orbiting around it at the desired distance; this tracking mode is called standoff tracking $[9,10]$. The guidance problem in cooperative standoff tracking can be divided into two aspects: relative distance control and inter-UAV angle control, which are realized by the lateral and longitudinal guidance law, respectively.

Lawrence proposed a guidance law based on the Lyapunov vector field guidance (LVFG) method [11]. In the study by Frew et al. [12], it is assumed that the target is moving at a constant velocity, and the LVFG has been proposed to improve the guidance accuracy of the relative distance; then, another Lyapunov function is used to solve the flight velocity command as the longitudinal guidance law of the UAV to control the inter-UAV angle. A weighted relative distance and inter-UAV angle error are used as the objective function, and the horizontal and vertical guidance commands for the next optimization step are searched by the method of model predictive control (MPC) [13]. This method not only needs a lot of calculation time but also still has some difficulties to achieve online applications. A cyclic pursuit for coordinated target tracking applications based on sliding mode control and a virtual leader is presented, but the guidance law is too complicated to achieve in practical projects [14]. A variant cycle of standoff tracking applications has also been investigated, while only fixed and uniform moving targets were considered for this method [15]. Cheng proposed a composite impact time control 
guidance law considering the problem of simultaneous attack against a ground target [16]. It is also necessary to consider the effect of disturbances, so the robust leaderfollower formation guidance laws are derived [17-19].

From the above previous work, we can conclude that the existing research results only study the static target instead of the moving target which limits the practical application of the guidance laws. To the best of the author's knowledge, few research results can consider both scenarios in a unified control framework. The developed results in this paper not only realize the objective of the tracking static target but also can track the moving target in a unified analytical control framework. In this paper, a new leader-follower formation tracking mode is proposed, which is based on the principle that a leader UAV is used to track a ground target in the standoff mode. Besides, when multiple follower UAVs track the leader UAV and are evenly distributed in a circle, the leader UAV and follower UAVs adopt the same tracking distance. As a result, the leader UAV maintains a certain distance from the ground target to avoid being exposed, and the follower UAVs can be closer to monitoring the ground target. The novelty of this paper lays in extending the practical application of standoff cooperative tracking of multi-UAVs not only for static targets but also for moving targets.

This paper is organized as follows. Section 1 describes the mathematical model of a ground target tracking problem. Section 2 provides the new guidance law of the leader UAV and mathematical stability proof. Section 3 provides the new guidance law of the follower UAVs and mathematical stability proof. The results of simulation are discussed in Section 4. The paper ends with some conclusions.

\section{Problem Formulation}

In order to develop guidance laws for the fixed-wing UAV that tracks a ground target, some assumptions have been stated. Firstly, the UAV is considered at a constant velocity and altitude over the ground. Furthermore, a separate inner loop (stabilization loop) and an outer loop (guidance loop) control approach are designed, similarly as in most applications [20-23].

In Figure 1, let $\rho$ denote the range between the UAV and the target, $\rho \geq 0$ and $\rho$ is bounded. The bearing angle $\chi \in[0,2 \pi)$ is defined as the angle between the UAV forward direction and the direction from the UAV to the target, which is measured counterclockwise. $\rho_{d}$ denotes the desired radius, and $v$ is the velocity of the $\mathrm{UAV}$.

The system dynamics can be described by the following kinematical model:

$$
\left\{\begin{array}{l}
\dot{x}=v \cos (\psi), \\
\dot{y}=v \sin (\psi), \\
\dot{\psi}=\omega, \\
\dot{v}=u,
\end{array}\right.
$$

where $[x, y]^{T}$ is the $2 \mathrm{D}$ location of the UAV, $\psi$ denotes the heading angle, $\omega$ and $u$ are the angular rate and

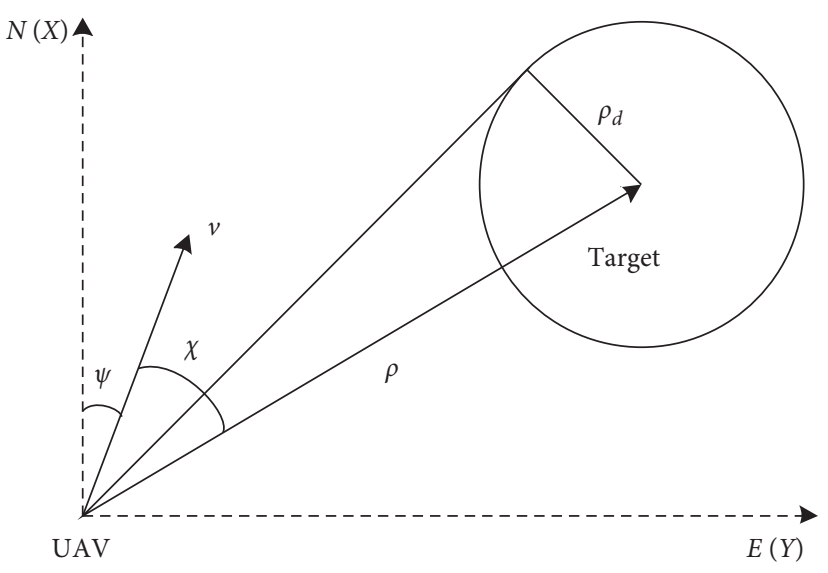

Figure 1: Geometry of tracking a stationary target.

acceleration as the control inputs, respectively, and $\left[x_{t}, y_{t}\right]^{T}$ denotes the 2D location of the target; then, the range $\rho=\sqrt{\left(x-x_{t}\right)^{2}+\left(y-y_{t}\right)^{2}}$. The objective is to design control input $\omega$ and usuch that $\rho \longrightarrow \rho_{d}$ as $t \longrightarrow \infty$.

The dynamics (1) can be rewritten as

$$
\left\{\begin{array}{l}
\dot{\rho}=-v \cos (\chi), \\
\dot{\chi}=\omega+\frac{v \sin (\chi)}{\rho}, \\
\dot{v}=u
\end{array}\right.
$$

In dynamics (2), the state variables are changed from $[x, y, \psi]^{T}$ to $[\rho, \chi, v]^{T}$. Furthermore, it can be seen that if the velocity of the UAV is constant, $\dot{\rho}$ and $\chi$ can be determined by eachother. When $\dot{\rho}=0, \chi=(\pi / 2)$ or $(3 \pi / 2)$, which represents the clockwise motion and counterclockwise motion of the UAV, respectively.

\section{Leader UAV Guidance Law Design}

Firstly, a guidance law of the leader UAV to track a static target is designed, and then, the guidance law is expanded to track a moving target.

3.1. Static Ground Target Tracking. In this paper, the guidance law for the leader UAV tracking a static ground target in the standoff mode is designed as

$$
\left\{\begin{array}{l}
\omega=k v \cos \chi-\frac{v \sin \chi}{\rho}-v\left(\rho-\rho_{d}\right), \\
u=0 .
\end{array}\right.
$$

Then, we give the following conclusion.

Theorem 1. Consider UAV dynamics in (2) subject to the guidance law in (3). If $k>0,\left(\rho_{d}, \pi / 2\right)^{T}$ is the asymptotically stable equilibrium point of the closed-loop system. 
Proof. consider the following candidate Lyapunov function:

$$
L_{1}=1-\sin \chi+\frac{1}{2}\left(\rho-\rho_{d}\right)^{2}
$$

It can be verified that $L_{1} \geq 0$, and $L_{1}=0$ only at $\left(\rho_{d}, \pi / 2\right)^{T}$.

Taking the derivative of $L_{1}$ yields that

$$
\dot{L}_{1}=-\cos \chi \cdot \dot{\chi}+\left(\rho-\rho_{d}\right) \cdot \dot{\rho} .
$$

With (2), we have

$$
\dot{L}_{1}=v \cos \chi \cdot\left(-\frac{\omega}{v}-\frac{\sin \chi}{\rho}-\rho+\rho_{d}\right) .
$$

Substituting $\omega$ into $\dot{L}_{1}$, we have

$$
\dot{L}_{1}=-k v \cos ^{2} \chi \text {. }
$$

Obviously, when $k>0$, for any $\chi$, there is $\dot{L}_{1} \leq 0$, and it can be concluded that $\dot{L}_{1}=0$ if and only if $\chi=(\pi / 2)$. Let $S=\left\{[\rho, \chi]^{T} \in R \times R \mid \chi=(\pi / 2)\right\}$; when $L_{1}=0$, we can obtain that $\rho=\rho_{d}$, and no other solution can stay identically in $S$ other than $\left(\rho_{d}, \pi / 2\right)^{T}$. According to LaSalle's invariance principle (Khalil, 2002), $\left(\rho_{d}, \pi / 2\right)^{T}$ is the asymptotically stable equilibrium point of the closed-loop system.

Remark. this Lyapunov function is well designed as its equilibrium point is $\chi=(\pi / 2), \rho=\rho_{d}$. By designing appropriate guidance laws, we can make $\dot{L}_{1} \leq 0$ till $L_{1}=0$, and the control objective can be realized.

3.2. Constant Velocity Ground Target Tracking. When the ground target moves at velocity $v_{t}$, the UAV kinematics' model can be written as

$$
\left\{\begin{array}{l}
\dot{\rho}=-v \cos \chi+v_{t} \cos \left(\psi-\psi_{t}+\chi\right), \\
\dot{\chi}=\omega+\frac{1}{\rho}\left(v \sin \chi-v_{t} \sin \left(\psi-\psi_{t}+\chi\right)\right), \\
\dot{v}=u
\end{array}\right.
$$

where the velocity vector of the UAV can be decomposed into relative velocity, and the target velocity vector (depicted in Figure 2)

$$
\vec{v}=\vec{v}_{m}+\vec{v}_{t}
$$

where $\vec{v}_{m}$ is the relative velocity vector. Then, the dynamics in (8) can be rewritten as the relative motion:

$$
\left\{\begin{array}{l}
\dot{\rho}=-v_{m} \cos \chi_{m} \\
\dot{\chi}_{m}=\omega_{m}+\frac{1}{\rho} v_{m} \sin \chi_{m} \\
\dot{v}_{m}=u_{m}
\end{array}\right.
$$

Velocity vector $v_{t}$ is constant when the target is in uniformly moving motion. Let $\vec{n}_{m}$ be the unit tangent

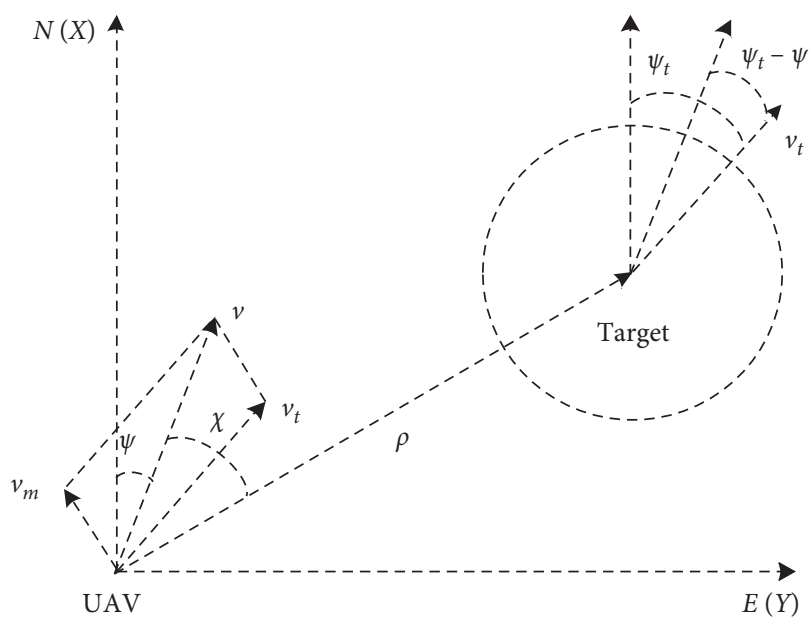

Figure 2: Geometry of tracking a moving target.

vector of $\vec{v}_{m}$ and $\vec{\omega}$ be the angular velocity vector corresponding to $\vec{v}$; taking the derivative of (9) yields that

$$
\vec{\omega} \times \vec{v}=\dot{v}_{m} \vec{n}_{m}+\vec{\omega}_{m} \times \vec{v}_{m} .
$$

The symbol " $x$ " denotes the cross product of vectors. Take the norm of both sides because $\dot{v}_{m}=(\vec{\omega} \times \vec{v}) \cdot \vec{n}_{m}$; thus, we can obtain that

$$
|\vec{\omega} \times \vec{v}|^{2}=\left|(\vec{\omega} \times \vec{v}) \cdot \vec{n}_{m}\right|^{2}+\left|\vec{\omega}_{m} \times \vec{v}_{m}\right|^{2} .
$$

Then, we get

$$
\begin{aligned}
\omega^{2} v^{2} & =\omega^{2} v^{2} \sin ^{2}\left(\psi-\psi_{m}\right)+\omega_{m}^{2} v_{m}^{2}, \\
\omega_{m}^{2} v_{m}^{4} & =\omega^{2}\left[v^{2} v_{m}^{2}-\left|\vec{v} \times \vec{v}_{m}\right|^{2}\right] .
\end{aligned}
$$

According to the parallelogram law of the vector, the area of the two triangles which are constituted by $\vec{v}$ and $\vec{v}_{t}$ and $\vec{v}$ and $\vec{v}_{m}$, respectively, are equal, that is,

$$
\left|\vec{v} \times \vec{v}_{t}\right|=\left|\vec{v} \times \vec{v}_{m}\right|
$$

Then, we can obtain that

$$
\omega_{m}^{2} v_{m}^{4}=\omega^{2}\left[v^{2} v_{m}^{2}-\left|\vec{v} \times \vec{v}_{t}\right|^{2}\right]
$$

We have

$$
\omega_{m}=\frac{v \sqrt{v_{m}^{2}-v_{t}^{2} \sin \left(\psi_{t}-\psi\right)}}{v_{m}^{2}} \omega .
$$

Finally, we can obtain the guidance law for tracking the constant velocity ground target:

$$
\left\{\begin{array}{l}
\omega=\frac{v_{m}^{2}\left[k v_{m} \cos \chi_{m}-\left(v_{m} \sin \chi_{m} / \rho\right)-v_{m}\left(\rho-\rho_{d}\right)\right]}{v \sqrt{v_{m}^{2}-v_{t}^{2} \sin ^{2}\left(\psi_{t}-\psi\right)}}, \\
u=0,
\end{array}\right.
$$

and the corresponding closed-loop system can be written as 


$$
\left\{\begin{array}{l}
\dot{\rho}=-v_{m} \cos \chi_{m} \\
\dot{\chi}=\frac{v_{m}^{2}\left[k v_{m} \cos \chi_{m}-\left(v_{m} \sin \chi_{m} / \rho\right)-v_{m}\left(\rho-\rho_{d}\right)\right]}{v \sqrt{v_{m}^{2}-v_{t}^{2} \sin ^{2}\left(\psi_{t}-\psi\right)}+\frac{1}{\rho} v_{m} \sin \chi_{m}} \\
\dot{v}=0 .
\end{array}\right.
$$

Then, we give the following conclusion.

Theorem 2. Consider UAV dynamics in (8) subject to the guidance law in (17). If $k>0,\left(\rho_{d},(\pi / 2)\right)^{T}$ is the asymptotically stable equilibrium point of the closed-loop system in (18).

Proof. consider the following candidate Lyapunov function:

$$
L_{2}=1-\sin \chi_{m}+\frac{1}{2}\left(\rho-\rho_{d}\right)^{2}
$$

Taking the derivative of $L_{2}$ yields that

$$
\dot{L}_{1}=-\cos \chi_{m} \cdot \dot{\chi}_{m}+\left(\rho-\rho_{d}\right) \cdot \dot{\rho} .
$$

Substitute (10) and (17) into $\dot{L}_{2}$, then

$$
\dot{L}_{2}=-k v \cos ^{2} \chi_{m} \text {. }
$$

It can be seen that if $k>0, \dot{L}_{2} \leq 0$ always exit and $\dot{L}_{2}=0$ only if $\chi_{m}(t)=(\pi / 2) \cdot \chi_{m}$ and $\rho(t)$ are both bounded and $\dot{L}_{2}(t)$ is also bounded according to Barbalat's lemma [20], when $\dot{L}_{2}(t) \longrightarrow 0, \chi_{m}(t) \longrightarrow(\pi / 2)$. Furthermore, because $\ddot{\chi}_{m}(t)$ is bounded, still according to Barbalat's lemma, we can conclude that when $\chi_{m}(t) \longrightarrow 0, \rho(t) \longrightarrow \rho_{d}$, and $\left(\rho_{d}, \pi / 2\right)^{T}$ is the asymptotically stable equilibrium point of the closed-loop system.

3.3. Variable Velocity Ground Target. When the ground target moves at variable velocity $v_{t}$, then $v$ and $v_{m}$ both are also variable, and equation (9) can be rewritten in the following scalar form:

$$
\left\{\begin{array}{l}
v \cos \psi=v_{m} \cos \psi_{m}+v_{t} \cos \psi_{t}, \\
v \sin \psi=v_{m} \sin \psi_{m}+v_{t} \sin \psi_{t} .
\end{array}\right.
$$

Taking the derivative of both sides' yields, one can have

$$
\left\{\begin{array}{l}
\dot{v} \cos \psi+v \dot{\psi} \cos \psi=\dot{v}_{m} \cos \psi_{m}+v_{m} \dot{\psi}_{m} \cos \psi_{m} \\
+\dot{v}_{t} \cos \psi_{t}+v_{t} \dot{\psi}_{t} \cos \psi_{t}, \\
\dot{v} \sin \psi+v \dot{\psi} \sin \psi=\dot{v}_{m} \sin \psi_{m}+v_{m} \dot{\psi}_{m} \sin \psi_{m} \\
+\dot{v}_{t} \sin \psi_{t}+v_{t} \dot{\psi}_{t} \sin \psi_{t} .
\end{array}\right.
$$

Solving the quadratic equation and eliminating $\dot{v}_{m}$, we can obtain the guidance law for tracking the variable velocity ground target:

$$
\left\{\begin{array}{l}
\omega=\frac{1}{v \cos \left(\psi-\psi_{m}\right)}\left(v_{m} \omega_{m}+v_{t} \omega_{t} \cos \left(\psi_{t}-\psi_{m}\right)\right), \\
u=\dot{v}_{t} \frac{\sin \left(\psi_{t}-\psi_{m}\right)}{\sin \left(\psi-\psi_{m}\right)}
\end{array}\right.
$$

where

$$
\omega_{m}=k v_{m} \cos \chi_{m}-\frac{v_{m} \sin \chi_{m}}{\rho}-v_{m}\left(\rho-\rho_{d}\right) .
$$

The corresponding closed-loop system can be written as

$$
\left\{\begin{array}{l}
\dot{\rho}=-v_{m} \cos \chi_{m}, \\
\dot{\chi}=\frac{1}{v \cos \left(\psi-\psi_{m}\right)}\left(v_{m} \omega_{m}+v_{t} \omega_{t} \cos \left(\psi_{t}-\psi_{m}\right)\right)+\frac{1}{\rho} v_{m} \sin \chi_{m}, \\
\dot{v}=\dot{v}_{t} \frac{\sin \left(\psi_{t}-\psi_{m}\right)}{\sin \left(\psi-\psi_{m}\right)} .
\end{array}\right.
$$

We can see that equations (26) and (18) are the same, thus still satisfy Theorem 2 for the variable velocity ground target.

\section{Follower UAV Guidance Law Design}

Consider $N$ follower UAVs in the formation problem, the $i$ th $(i=1,2, \ldots, N)$ UAV's Dubins model can be obtained as follows:

$$
\left\{\begin{array}{l}
\dot{x}_{i}=v_{i} \cos \left(\psi_{i}\right) \\
\dot{y}_{i}=v_{i} \sin \left(\psi_{i}\right), \quad(i=1,2, \ldots, N) . \\
\dot{\psi}_{i}=\omega_{i} \\
\dot{v}_{i}=u_{i}
\end{array}\right.
$$

The geometry between the follower UAVs and the leader $\mathrm{UAV}$ is depicted in Figure 3.

In this paper, the follower UAVs are required to maintain a circular formation with the centre at the leader UAV and hold equal angular separation. Meanwhile, the follower UAV's velocity and heading angle should gradually converge to the leader UAV's. The relative motion can be written as

$$
\left\{\begin{array}{l}
\dot{\rho}_{i}=v_{i} \cos \left(\theta_{i}-\psi_{i}\right)-v_{0} \cos \left(\theta_{i}-\psi_{0}\right), \\
\dot{\theta}_{i}=\frac{-v_{i} \sin \left(\theta_{i}-\psi_{i}\right)+v_{0} \sin \left(\theta_{i}-\psi_{0}\right)}{\rho_{i}},
\end{array}\right.
$$

where the subscript ' 0 ' denotes the leader UAV.

The follower UAV's angular rate control is used to carry out distance and heading angle tracking for the leader UAV, while formation's inter-UAV angle control and velocity tracking are performed by velocity control. We use such a 


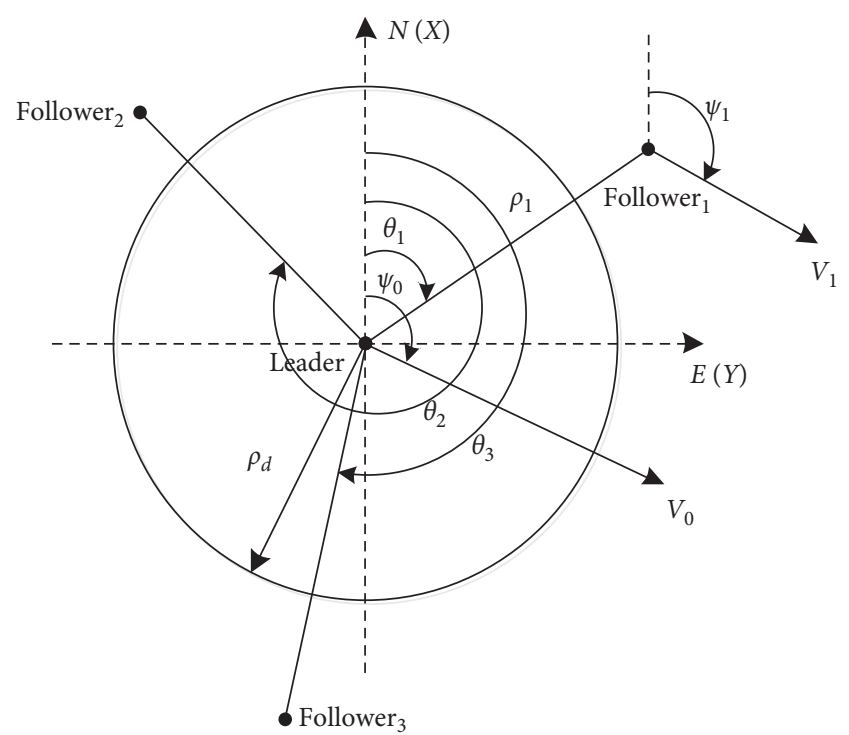

Figure 3: Geometry of follower UAVs to track the leader UAV.

communication topology, that is, the follower UAVs can perceive the state of the leader UAV, and a circular communication structure is implemented among the follower UAVs.

4.1. Angular Rate Guidance Law Design. In this paper, the following angular rate guidance law is designed for the $i$ th follower UAV:

$$
\omega_{i}=\dot{\psi}_{0}-\frac{\left(\rho_{i}-\rho_{d}\right)}{\left(\psi_{i}-\psi_{0}\right)} \dot{\rho}_{i}-k_{\omega}\left(\psi_{i}-\psi_{0}\right) .
$$

Then, we give the following conclusion.

Theorem 3. Consider the UAV formation dynamics in (28) subject to the guidance law in (29). If $k_{\omega}>0$, the distance between the follower UAV and the leader UAV will gradually converge to $\rho_{d}$, and the heading angle of the follower UAV will also gradually converge to the heading angle of the leader $U A V$.

Proof. consider the following candidate Lyapunov function:

$$
L_{\omega}=\frac{1}{2} \sum_{i=1}^{N}\left[\left(\rho_{i}-\rho_{d}\right)^{2}+\left(\psi_{i}-\psi_{0}\right)^{2}\right] .
$$

Taking the derivative yields that

$$
\dot{L}_{\omega}=\sum_{i=1}^{N}\left[\left(\rho_{i}-\rho_{d}\right) \cdot \dot{\rho}_{i}+\left(\psi_{i}-\psi_{0}\right) \cdot\left(\dot{\psi}_{i}-\dot{\psi}_{0}\right)\right],
$$

where $\dot{\psi}_{i}=\omega_{i}$, and

$$
\omega_{i}=\dot{\psi}_{0}-\frac{\left(\rho_{i}-\rho_{d}\right)}{\left(\psi_{i}-\psi_{0}\right)} \cdot \dot{\rho}_{i}-k_{\omega} \cdot\left(\psi_{i}-\psi_{0}\right),
$$

and we get

$$
\dot{L}_{\omega}=\sum_{i=1}^{N}\left(-k_{\omega} \cdot\left(\psi_{i}-\psi_{0}\right)^{2}\right) \leq 0 .
$$

So $\psi_{i}-\psi_{0}$ is bounded, as $\dot{L}_{\omega}$ is uniformly continuous, and $\left(\psi_{i}-\psi_{0}\right) \longrightarrow 0$ according to Barbalat's lemma. Furthermore, as $\dot{\psi}_{i}-\dot{\psi}_{0}$ is uniformly continuous, we have $\left(\dot{\psi}_{i}-\dot{\psi}_{0}\right) \longrightarrow 0$ according to Barbalat's lemma; then, we can conclude that $\rho_{i} \longrightarrow \rho_{d}$.

4.2. Velocity Guidance Law Design. In this paper, the following velocity guidance law is designed for the $i$ th follower UAV:

$$
u_{i}=\dot{v}_{0}-\frac{(\Delta \theta-(2 \pi / N))}{\left(v_{i}-v_{0}\right)} \Delta \dot{\theta}-k_{v}\left(v_{i}-v_{0}\right) .
$$

Then, we give the following conclusion.

Remark. it is easy to find that the Lyapunov function $L_{\omega}$ is bounded, so we can conclude that $\left(\psi_{i}-\psi_{0}\right) \longrightarrow 0$ and $\left(\rho_{i}-\right.$ $\left.\rho_{d}\right) \longrightarrow 0$ simultaneously according to Barbalat's lemma. In practice, even if $\left(\psi_{i}-\psi_{0}\right)=0$, it is not an attractor which does not affect the convergence of the whole guidance system. It is the same for the case of $\left(v_{i}-v_{0}\right) \longrightarrow 0$. Hence, we can conclude that there exists no problem of singular values.

Theorem 4. Consider the UAV formation dynamics in (28) subject to the guidance law in (34); if $k_{v}>0$, the inter-UAV angle between follower UAVs will gradually converge to $(2 \pi / N)$, and the velocity of the ith follower UAV will gradually converge to the velocity of the leader UAV.

Proof. consider the following candidate Lyapunov function:

$$
L_{u}=\frac{1}{2} \sum_{i=1}^{N}\left[\left(\Delta \theta_{i}-\frac{2 \pi}{N}\right)^{2}+\left(v_{i}-v_{0}\right)^{2}\right] \text {. }
$$

Taking the derivative yields that

$$
\dot{L}_{u}=\sum_{i=1}^{N}\left[\left(\Delta \theta_{i}-\frac{2 \pi}{N}\right) \cdot \Delta \dot{\theta}_{i}+\left(v_{i}-v_{0}\right) \cdot\left(\dot{v}_{i}-\dot{v}_{0}\right)\right],
$$

where $\dot{v}_{i}=u_{i}$, and

$$
u_{i}=\dot{v}_{0}-\frac{\left(\Delta \theta_{i}-(2 \pi / N)\right)}{\left(v_{i}-v_{0}\right)} \Delta \dot{\theta}_{i}-k_{v}\left(v_{i}-v_{0}\right),
$$

and we get

$$
\dot{L}_{u}=\sum_{i=1}^{N}\left(-k_{v} \cdot\left(v_{i}-v_{0}\right)^{2}\right) \leq 0 .
$$

Similar with Theorem 3, we can also conclude that $\Delta \theta_{i} \longrightarrow(2 \pi / N)$ and $v_{i} \longrightarrow v_{0}$.

\section{Simulation Results}

In this section, some simulation results are presented in order to demonstrate the effectiveness of the proposed 


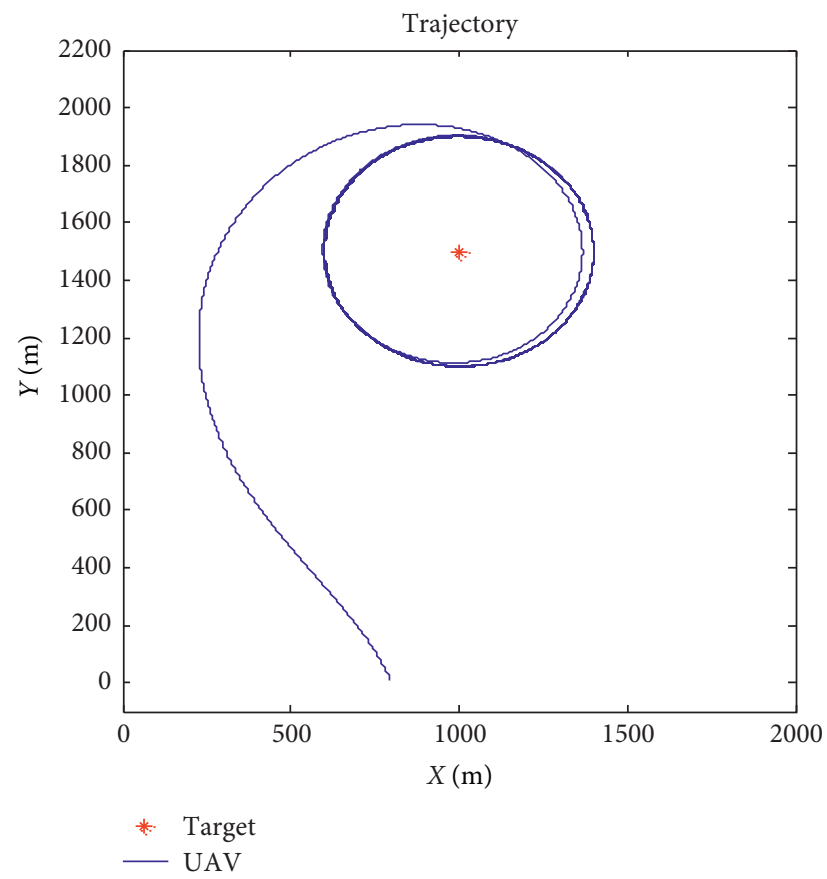

Figure 4: Trajectory of tracking a static target.

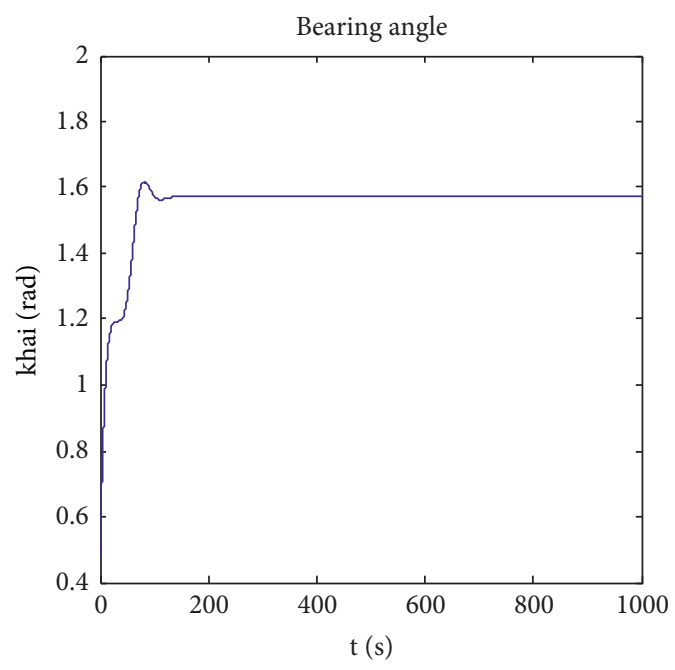

(a)

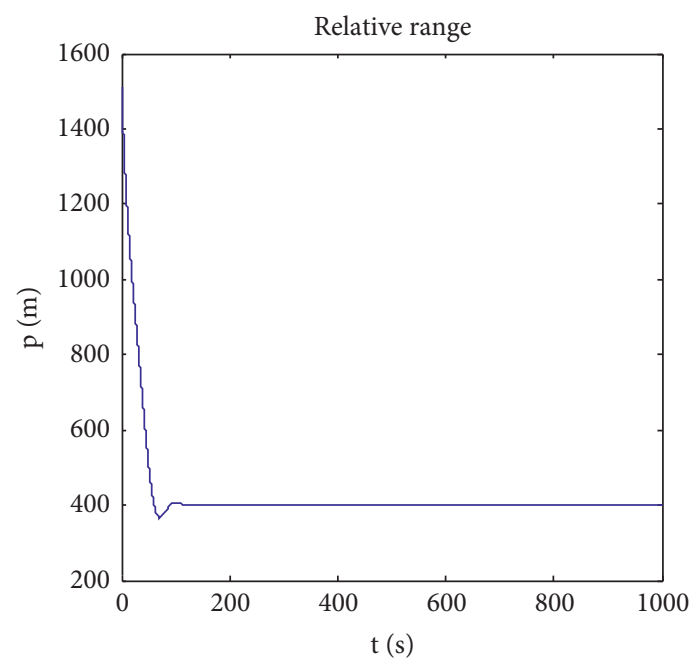

(b)

FIGURE 5: Bearing angle and relative range of tracking a static target. (a) Bearing angle. (b) Relative range.

leader-follower formation guidance laws. In these simulation cases, we consider a 4-UAVs' formation as an example, $\mathrm{UAV} \# 0$ is the leader UAV and UAV \#1-UAV \#3 are the follower UAVs. The ground target motion is considered as stationary, linear with constant velocity, and linear with variable velocity.

Firstly, the simulation applies the leader UAV to track a ground target and then the UAV formation to track the ground target in the same way.
5.1. Track a Ground Target Using One UAV. The initial states of the UAV are set as follows:

(i) Position coordinate: $(800,0)$

(ii) Heading angle: $-60^{\circ}$

(iii) Cruising velocity: $45 \mathrm{~m} / \mathrm{s}$

(iv) Maximum heading angular rate: $0.1 \mathrm{rad} / \mathrm{s}$

The initial states of the ground target are set as follows: 


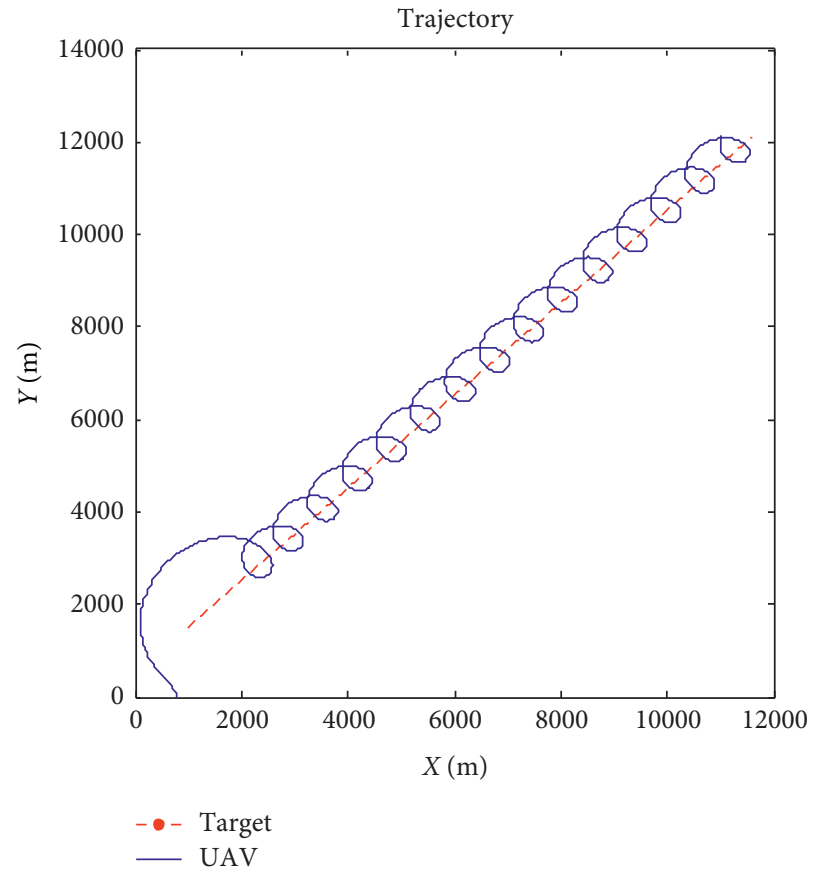

Figure 6: Trajectory of tracking a constant velocity target.

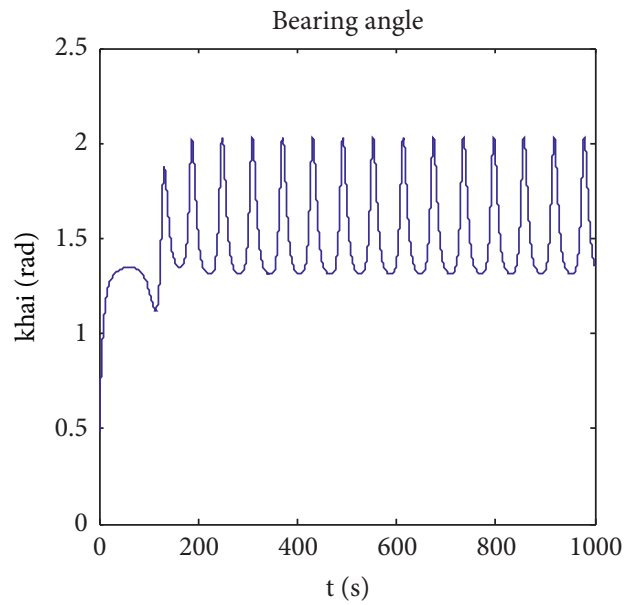

(a)

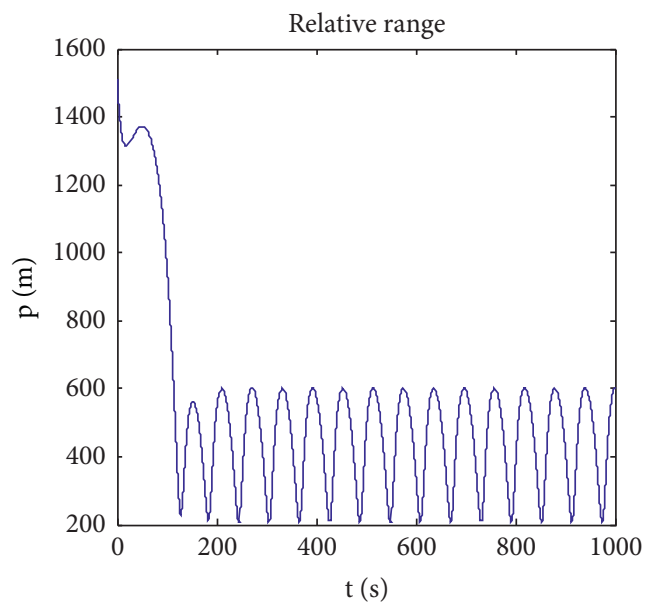

(b)

Figure 7: Bearing angle and relative range of tracking a constant velocity target. (a) Bearing angle. (b) Relative range.

(i) Position coordinate: $(1000,1500)$

(ii) Heading angle: $30^{\circ}$

(iii) Velocity: $15 \mathrm{~m} / \mathrm{s}$

The gain is chosen as $k=0.0025$.

5.1.1. Static Ground Target. Figure 4 shows that the trajectory of standoff tracking of one UAV around the static target, and it can be seen that the trajectory of the UAV converges to the circle surrounding the target under the proposed guidance law. Figure 5 shows the changes of the bearing angle and relative range.
5.1.2. Constant Velocity Ground Target. Figure 6 shows the standoff tracking trajectory of the UAV when the target moves with constant velocity. It can be seen that the trajectory of the UAV is the combination of circular motion and linear motion. The changes of the bearing angle and relative range during the process of the convergence are shown in Figure 7.

5.1.3. Variable Velocity Ground Target. The velocity of the ground target is chosen as

$$
v_{t}=12+2 \times \sin \left(\frac{t}{10}\right) .
$$




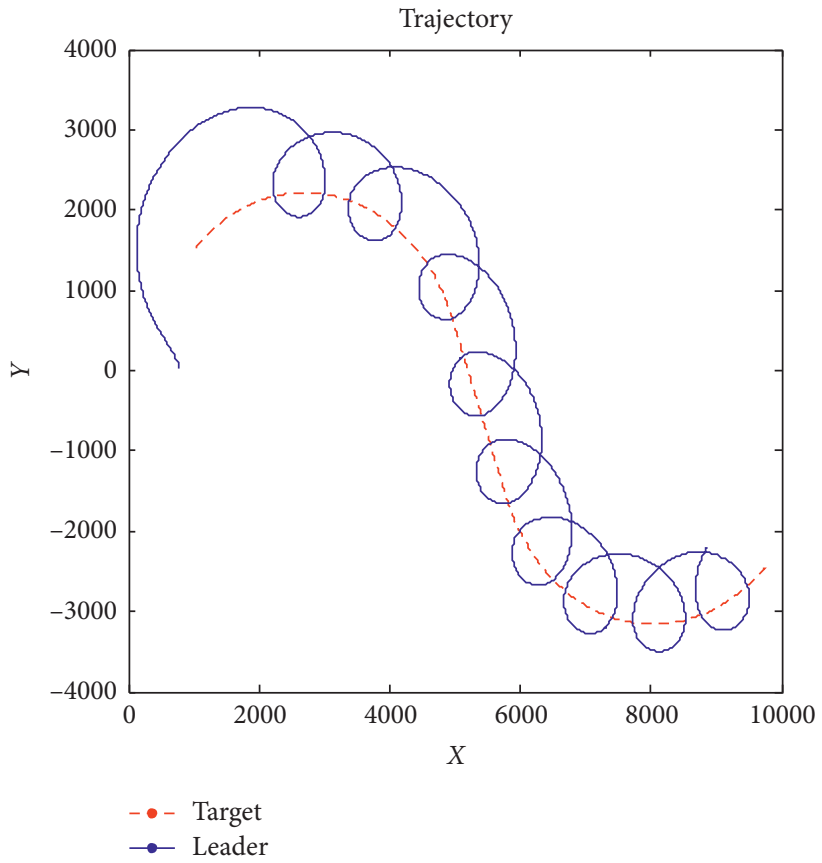

Figure 8: Trajectory of tracking a variable velocity target.

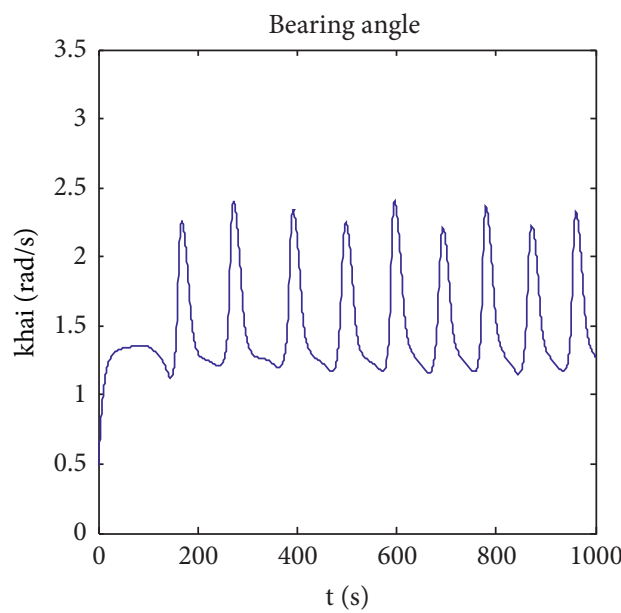

(a)

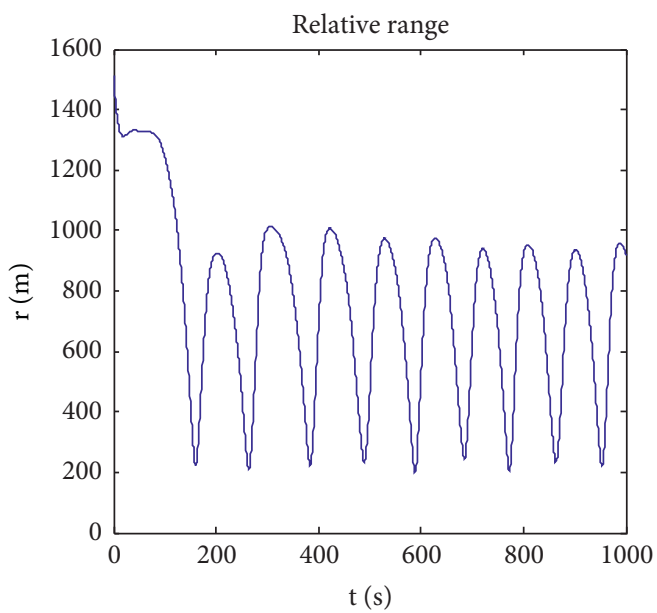

(b)

FIGURE 9: Bearing angle and relative range of tracking a variable velocity target. (a) Bearing angle. (b) Relative range.

The angular velocity of the ground target is set as

$$
\dot{\psi}_{t}(t)=\left\{\begin{array}{lc}
-0.005, & t<400, \\
0, & 400 \leq t \leq 600, \\
0.005, & t>600 .
\end{array}\right.
$$

In this scenario, the target moves with the sine trajectory. It can be seen that the proposed guidance law can also realize the standoff tracking of the target. The trajectory of the UAV and the ground target are shown in Figure 8, and the convergence curves of the bearing angle and relative range are shown in Figure 9.
From Figures 4 to 9, it can be seen that whether the target is still or moving, and the UAV always can track the ground target well in the standoff mode.

5.2. Track a Ground Target Using Formation UAVs. The initial states of the UAV \#1 are set as follows:

(i) Position coordinate: $(150,-1000)$

(ii) Heading angle: $-30^{\circ}$

(iii) Cruising velocity: $40.5 \mathrm{~m} / \mathrm{s}$

(iv) Maximum heading angular rate: $0.1 \mathrm{rad} / \mathrm{s}$ 


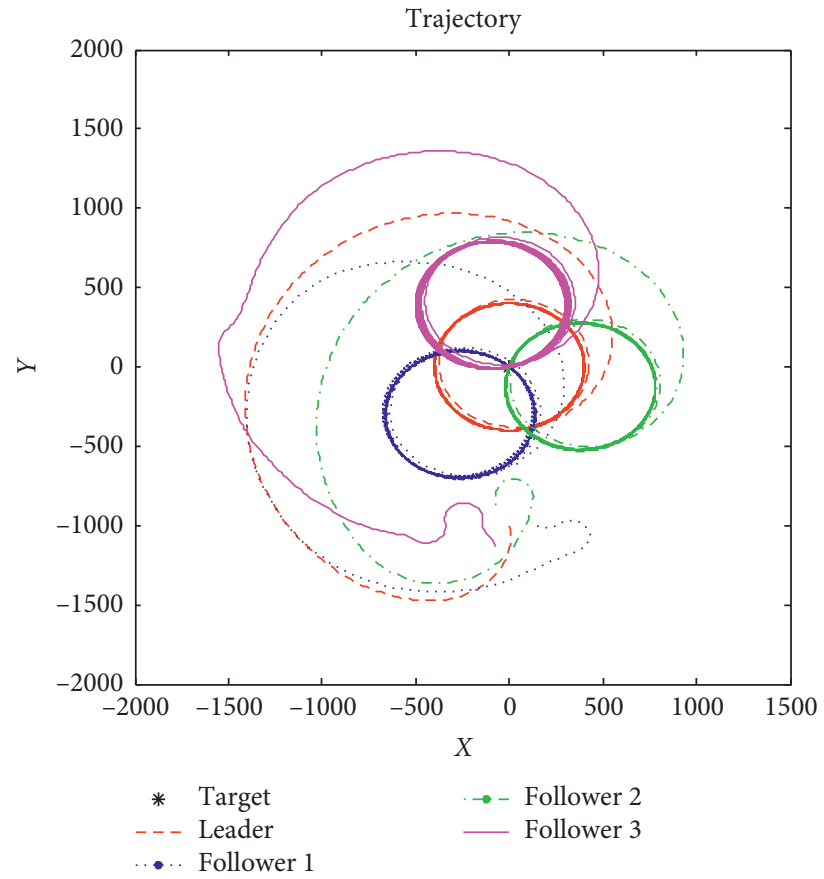

FIgURE 10: Trajectory of cooperative tracking a static target.

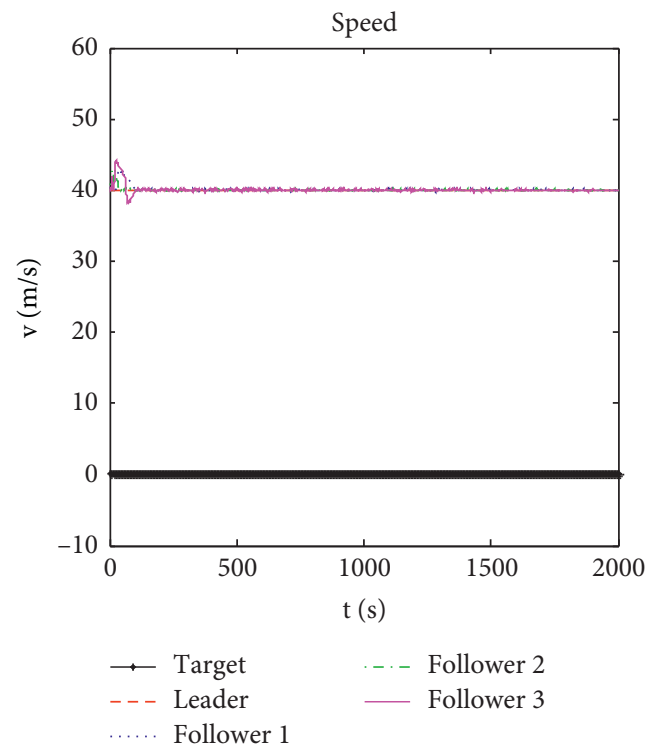

(a)

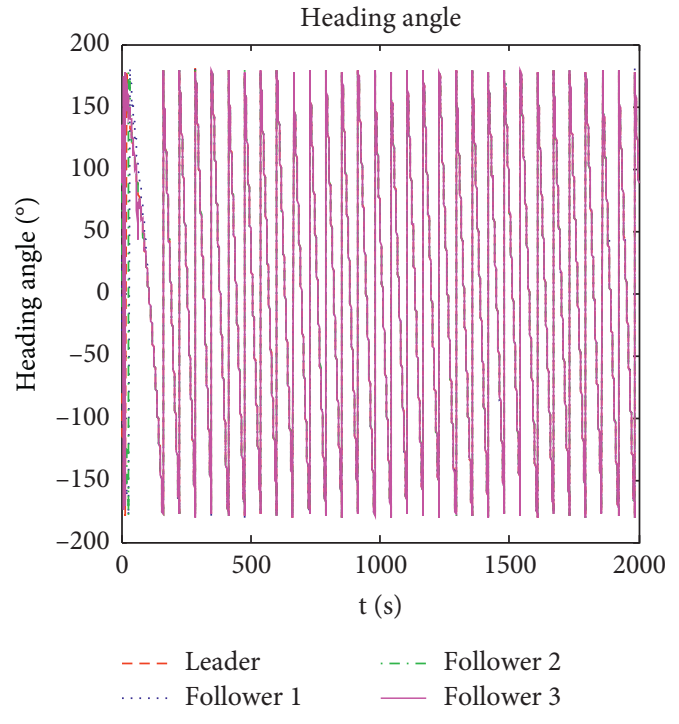

(b)

FIGURE 11: Velocities and heading angles of cooperative tracking a static target. (a) Velocities of target and followers. (b) Heading angles of all UAVs.

The initial states of the UAV \#2 are set as follows:
(i) Position coordinate: $(-75,-870)$
(ii) Heading angle: $80^{\circ}$
(iii) Cruising velocity: $41 \mathrm{~m} / \mathrm{s}$
(iv) Maximum heading angular rate: $0.1 \mathrm{rad} / \mathrm{s}$

The initial states of the UAV \#3 are set as follows:

(i) Position coordinate: $(-75,-1130)$

(ii) Heading angle: $100^{\circ}$

(iii) Cruising velocity: $40.5 \mathrm{~m} / \mathrm{s}$

(iv) Maximum heading angular rate: $0.1 \mathrm{rad} / \mathrm{s}$ 


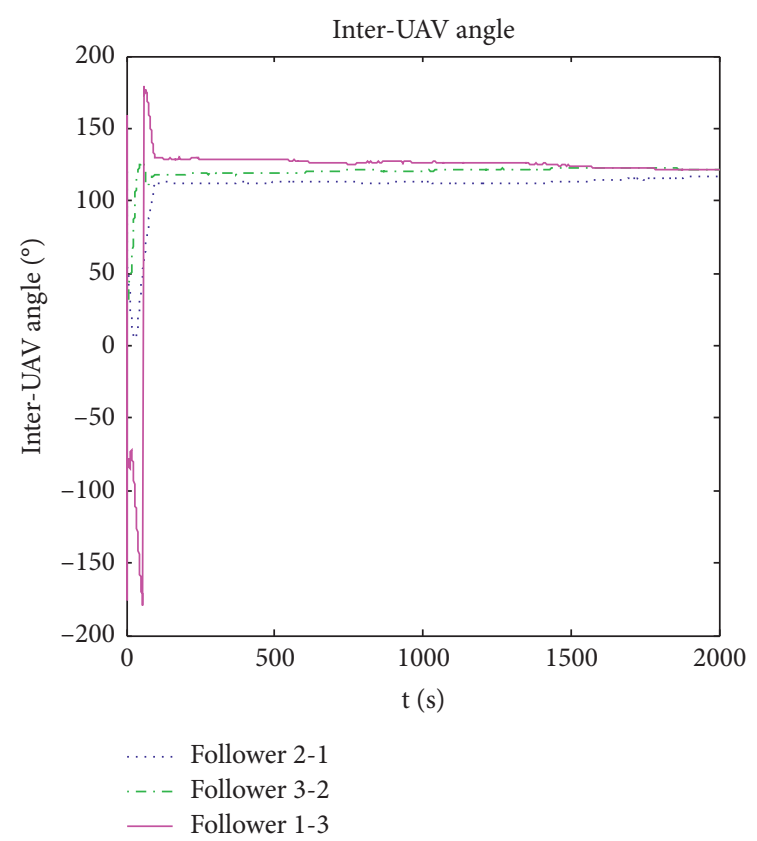

(a)

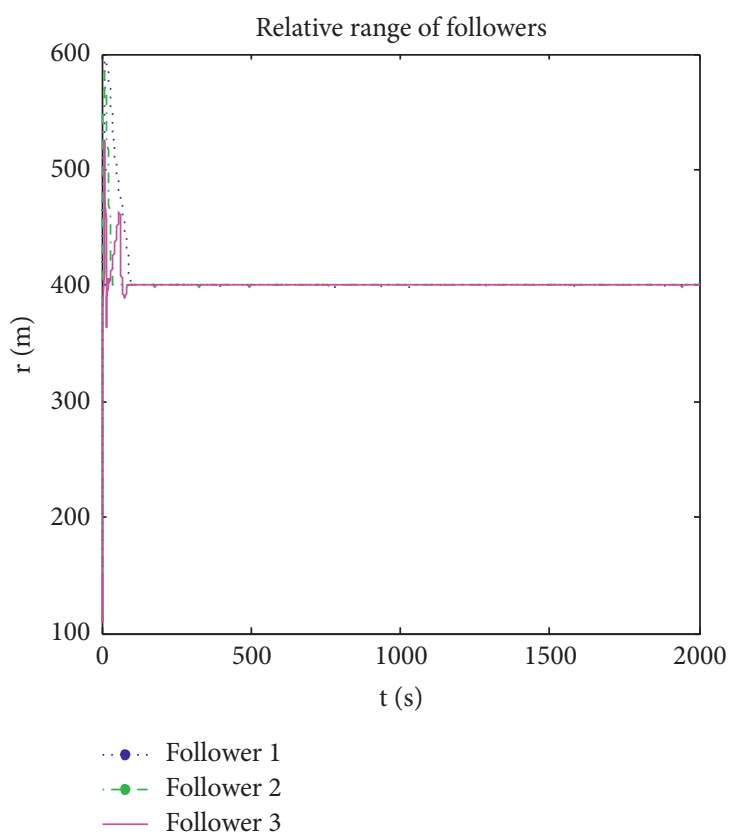

(b)

FIGURE 12: Inter-UAV angles and relative ranges of cooperative tracking a static target. (a) Inter-UAV angles between followers. (b) Relative ranges between leader and follower UAVs.

The gains are chosen as $k_{v}=1.28$ and $k_{\omega}=1.2$.

5.2.1. Static Ground Target. Under the proposed guidance laws, the multi-UAVs can converge to the desired standoff trajectories when the target is static. The trajectories of the UAVs' formation are shown in Figure 10, the velocities and heading angles of the formation are shown in Figure 11, and the inter-UAV angles and relative range between leader and follower UAVs are shown in Figure 12.

5.2.2. Constant Velocity Ground Target. As shown in Figure 13, the target moves with constant velocity and UAVs can accomplish the objective of standoff tracking, and the changes of the velocities and heading angles are shown in Figure 14, and the inter-UAV angle and relative range between leader and follower UAVs are shown in Figure 15.

5.2.3. Variable Velocity Ground Target. In this scenario, the target moves with the sine trajectory. The proposed guidance laws can also guarantee the convergence of the formation. The trajectories of the UAVs are shown in Figure 16, the changes of the velocities and heading angles are shown in Figure 17, and the inter-UAV angles and relative ranges between the leader and follower UAVs are shown in Figure 18.

From Figures 10 to 18, it can be seen that regardless of the ground target is static or moving, the desired formation always can be achieved, the follower UAVs' velocities and heading angles gradually converge to the leader, and the followers always can track the ground target well.

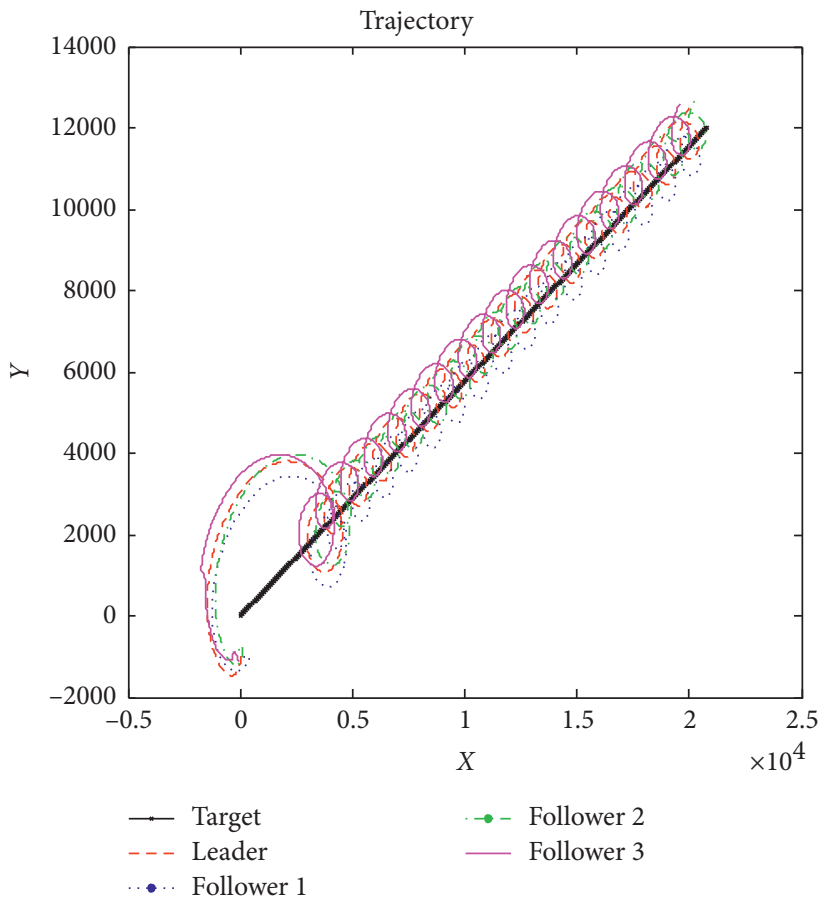

FIGURE 13: Trajectory of cooperative tracking a constant velocity target.

5.3. Simulation Analysis and Comparison. In order to verify the tracking performance, we adopt the well-known LVFG guidance algorithm proposed in Ref. [12] to run the same simulation case of variable velocity ground target tracking. We choose the tracking performance of UAV \#1 as an example. 


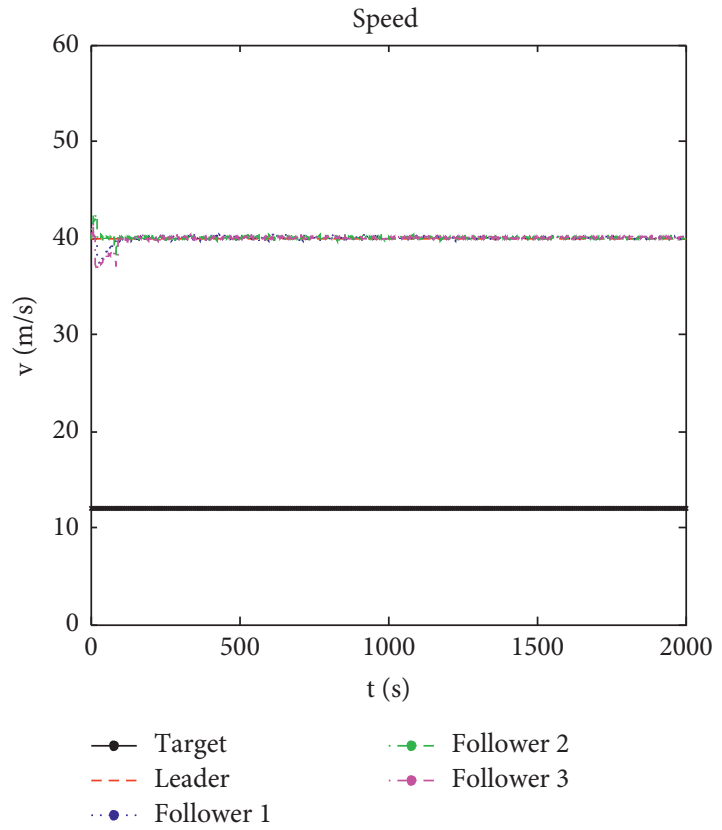

(a)

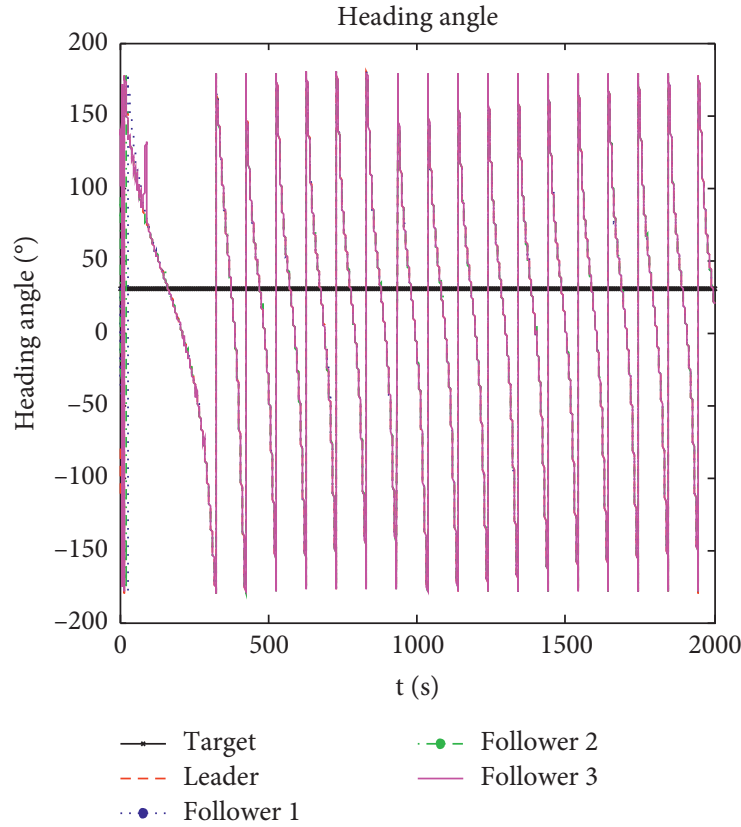

(b)

FIGURE 14: Velocities and heading angle of cooperative tracking a constant velocity target. (a) Velocities of target and followers. (b) Heading angles of all UAVs.

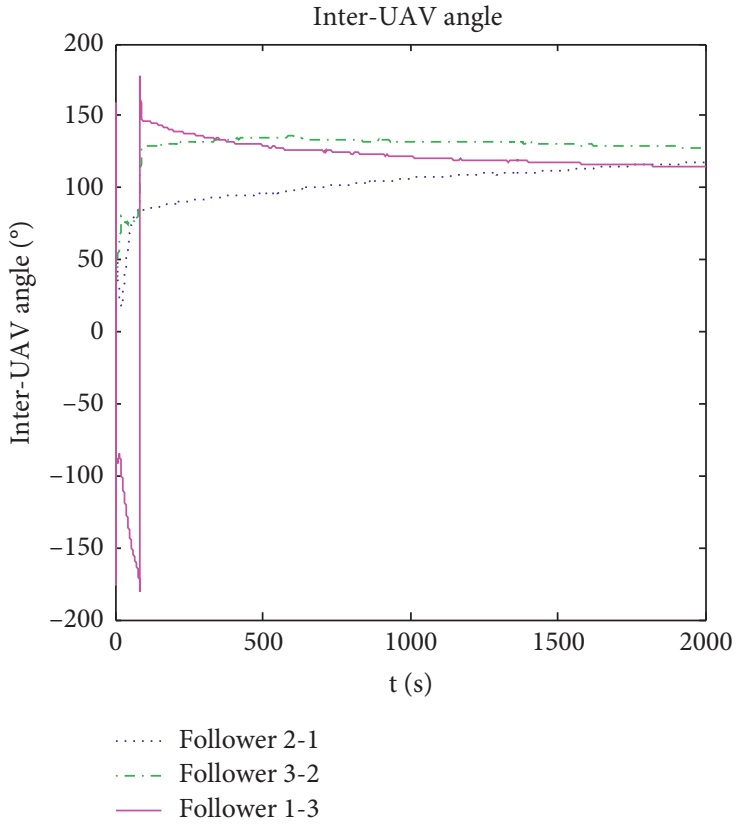

(a)

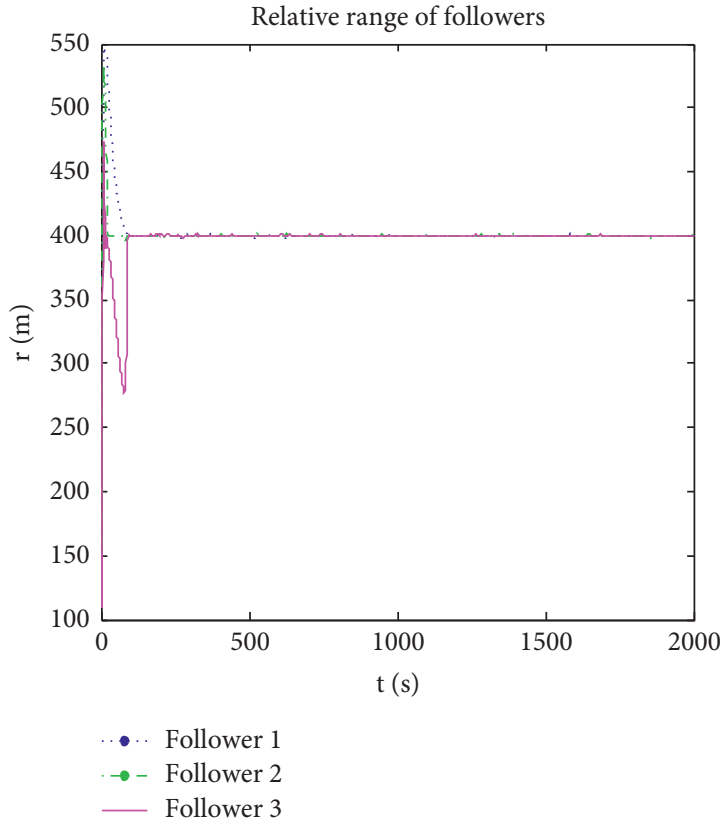

(b)

FIGURE 15: Inter-UAV angle and relative range of cooperative tracking a constant velocity target. (a) Inter-UAV angles between follower UAVs. (b) Relative range between leader and follower UAVs. 


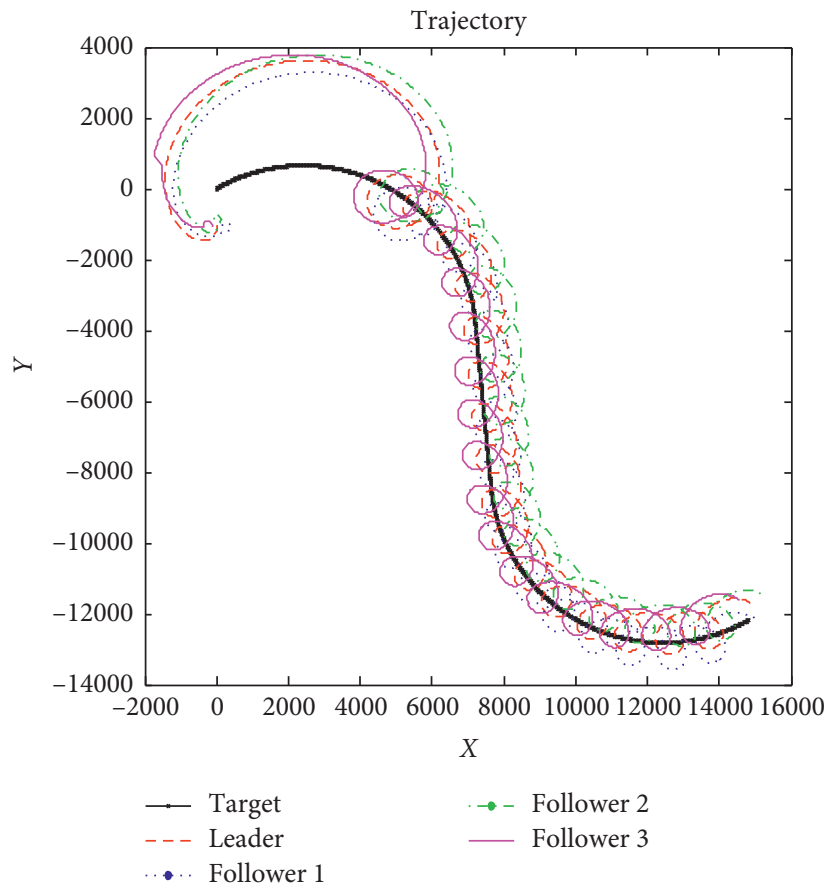

FIGURE 16: Trajectory of cooperative tracking a variable moving target.

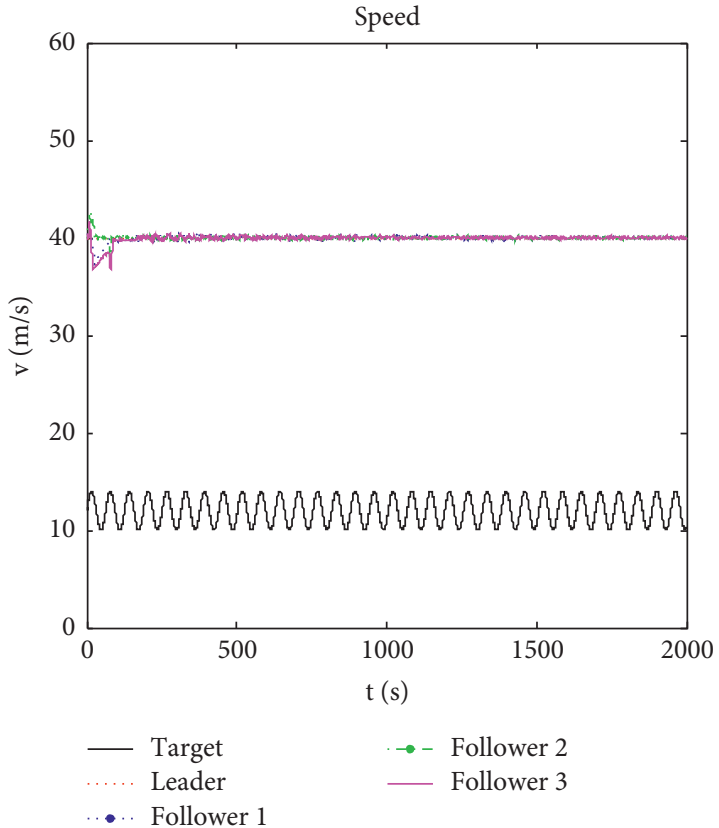

(a)

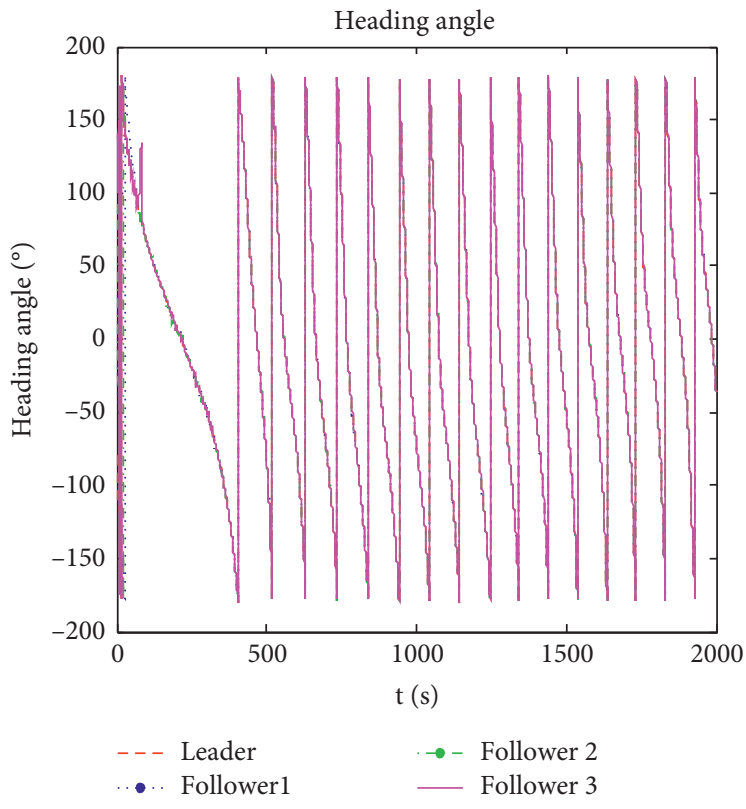

(b)

Figure 17: Velocities and heading angles of cooperative tracking a variable velocity target. (a) Velocity of target and followers. (b) Heading angles of all UAVs.

The velocity of $U A V \# 1$ and relative range between UAV $\# 1$ and the ground target using proposed method in this paper and Ref. [12] are shown in Figure 19.
When other performances are relative equivalent, from Figure 19, the proposed guidance law in this paper makes the velocity and heading angle of the follower UAVs always be 


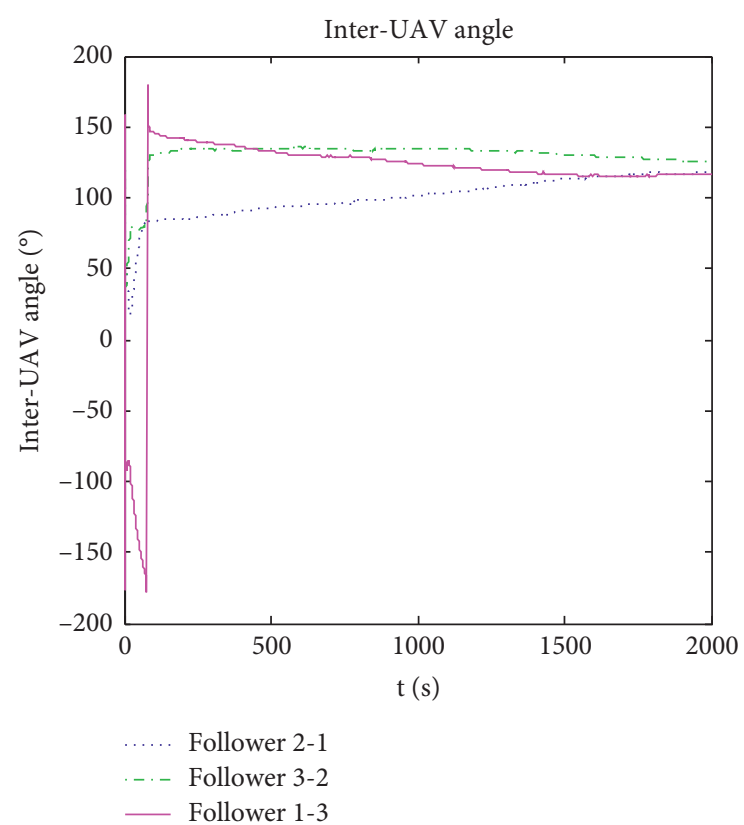

(a)

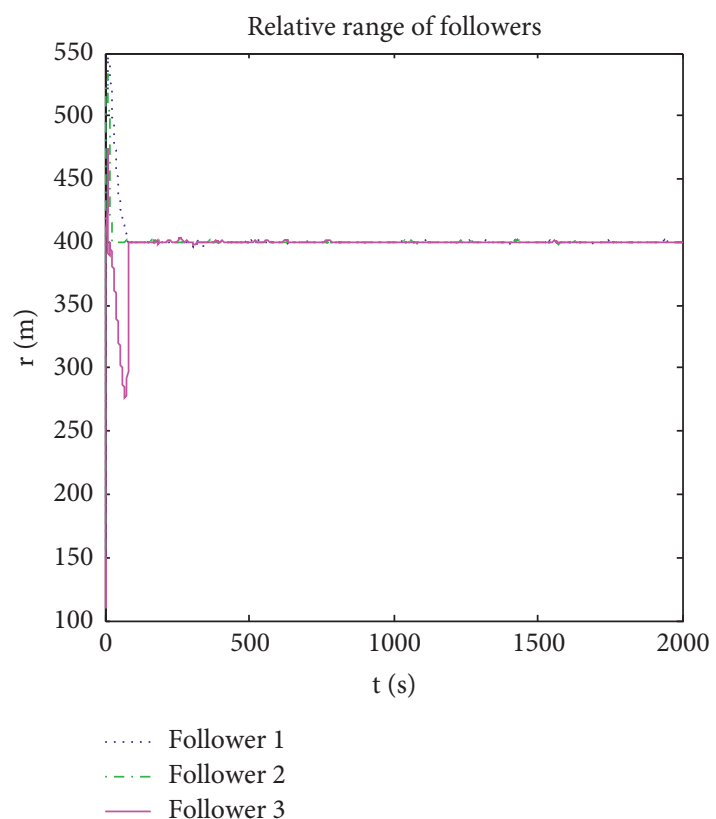

(b)

FIGURE 18: Inter-UAV angles and relative ranges of cooperative tracking a variable velocity target. (a) Inter-UAV angles between follower UAVs. (b) Relative ranges between leader and follower UAVs.

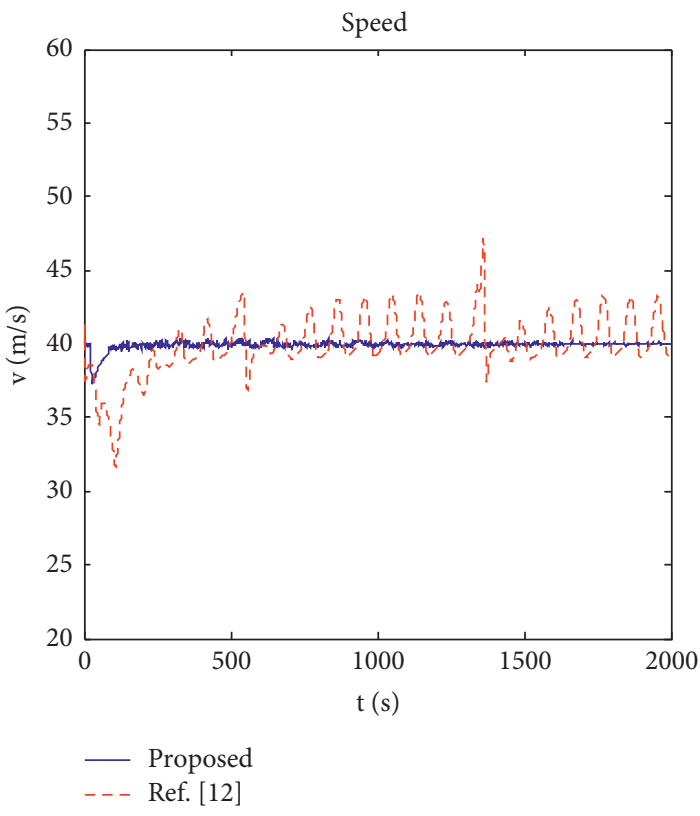

(a)

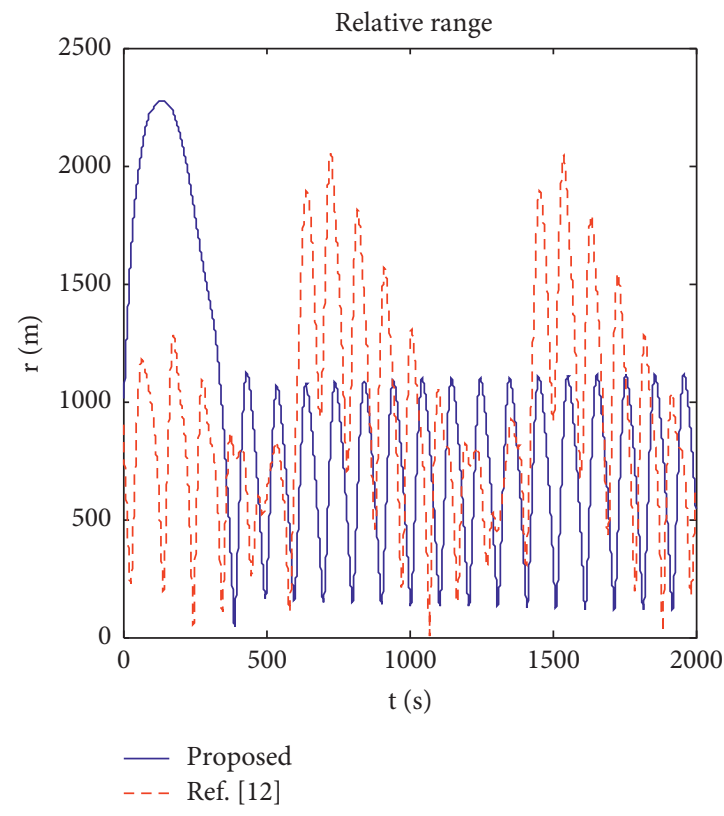

(b)

FIgURE 19: Velocity of UAV \#1 and relative range. (a) Velocity of UAV \#1. (b) Relative range.

consistent with the leader UAV, thus the convergence characteristics of velocity and relative range of the UAV \#1 are better than the results in Ref. [12].

\section{Conclusion}

In this paper, a new leader-follower formation tracking scheme is proposed, which is based on the principle that a leader UAV is applied to track a ground target in the standoff mode, while multiple follower UAVs to track the leader UAV and are evenly distributed in a circle. As a result, the leader UAV maintains a certain distance to the ground target to avoid being exposed, while the follower UAVs can be closer to monitoring the ground target. The stabilities of the new guidance laws are proved using Lyapunov functions. Numerical simulations of a 4 -UAVs' formation show that the new leader-follower 
formation can track the static and moving targets well, and its performance is better than the well-known classic LVFG algorithm [24].

\section{Data Availability}

The raw/processed data required to reproduce these findings cannot be shared at this time as the data also forms part of an ongoing study.

\section{Conflicts of Interest}

The authors declare that there are no conflicts of interest.

\section{Acknowledgments}

The work was supported by National Natural Science Foundation of China 61903192 and Natural Science Foundation of Jiangsu Province BK20190402.

\section{References}

[1] M. Wu and Y. Fei, Flight Control System, pp. 46-64, Beijing University of Aeronautics and Astronautics Press, Beijing, China, 2006.

[2] S. Rathinam, Z. W. Kim, and R. Sengupta, "Vision-based monitoring of locally linear structures using an unmanned aerial vehicle," Journal of Infrastructure Systems, vol. 14, no. 1, pp. 52-63, 2008.

[3] Y. Wang, H. Pu, D. Wang, and W. Jiang, "Unmanned aerial vehicles cooperative path planning for ground target tracking via chemical reaction optimization," Scientia Sinica Technologica, vol. 45, no. 6, pp. 583-594, 2015.

[4] T. Betts, "Survey of numerical methods for trajectory optimization," Journal of Guidance, Control, and Dynamics, vol. 21, no. 2, pp. 193-207, 2008.

[5] V. N. Dobrokhodov, I. I. Kaminer, K. D. Jones, and R. Ghabcheloo, "Vision-based tracking and motion estimation for moving targets using small UAVs," in Proceedings of the 2006 American Control Conference, Minneapolis, MN, USA, June 2006.

[6] Z. W. Kim and R. Sengupta, "Target detection and position likelihood using an aerial image sensor," in Proceedings of the 2008 IEEE International Conference on Robotics and Automation, Pasadena, CA, USA, May 2008.

[7] T.-H. Kim and T. Sugie, "Cooperative control for targetcapturing task based on a cyclic pursuit strategy," Automatica, vol. 43, no. 8, pp. 1426-1431, 2007.

[8] S. Daingri and A. Sinha, "nonlinear cyclic pursuit based cooperative target monitoring," in Proceedings of the International symposium on distributed autonomous robotic systems, Baltimore, MD, USA, November 2012.

[9] L. F. Bertuccelli and J. P. How, "Search for dynamic targets with uncertain probability maps," in Proceedings of the 2006 IEEE American Control Conference, Minneapolis, MN, USA, June 2006.

[10] Z. Li and N. Hovakimyan, "Vision-based target tracking and motion estimation using a small UAV," in Proceedings of the 2010 IEEE conference on Decision and Control, Atlanta, GA, USA, December 2010.

[11] D. A. Lawrence and E. W. Frew, "Lyapunov vector fields for autonomous unmanned aircraft flight control," Journal of
Guidance Control and Dynamics, vol. 31, no. 5, pp. 1220-1229, 2012.

[12] E. W. Frew, D. A. Lawrence, and S. Morris, "Coordinated standoff tracking of moving targets using Lyapunov guidance vector fields," Journal of Guidance, Control, and Dynamics, vol. 31, no. 2, pp. 290-306, 2008.

[13] S. Kim, H. Oh, and A. Tsourdos, "Nonlinear model predictive coordinated standoff tracking of a moving ground vehicle," Journal of Guidance, Control, and Dynamics, vol. 36, no. 2, pp. 557-566, 2013.

[14] M. Zhang and H. Liu, "Cooperative tracking a moving target using multiple fixed-wing UAVs," Journal of Intelligent and Robotic Systems, vol. 81, no. 3, pp. 505-529, 2018.

[15] A. Hashemi, Y. Cao, and D. W. Casbeer, "Unmanned aerial vehicle circumnavigation using noisy range-based measurements without global positioning system information," Journal of Dynamic Systems Measurement \& Control, vol. 137, no. 3, pp. 1-10, 2015.

[16] Z. Cheng, B. Wang, L. Liu, and Y. Wang, "A composite impact-time-control guidance law and simultaneous arrival," Aerospace Science and Technology, vol. 80, pp. 403-412, 2018.

[17] N. Zhang, W. Gai, G. Zhang, and J. Zhang, "An active disturbance rejection control guidance law based collision avoidance for unmanned aerial vehicles," Aerospace Science and Technology, vol. 77, pp. 658-669, 2018.

[18] L. He, P. Bai, X. Liang, J. Zhang, and W. Wang, "Feedback formation control of UAV swarm with multiple implicit leaders," Aerospace Science and Technology, vol. 72, pp. 327$334,2018$.

[19] X. Ai and J. Yu, "Flatness-based finite-time leader-follower formation control of multiple quadrotors with external disturbances," Aerospace Science and Technology, vol. 92, pp. 20-33, 2019.

[20] Y. Cao, "UAV circumnavigating an unknown target under a GPS-denied environment with range-only measurements," Automatica, vol. 55, pp. 150-158, 2014.

[21] Y. Cao, "UAV circumnavigating an unknown target using range measurement and estimated range rate," in Proceedings of the 2014 IEEE American Control Conference, Portland, OR, USA, June 2014.

[22] M. Varga, J.-C. Zufferey, G. H. M. Heitz, and D. Floreano, "Evaluation of control strategies for fixed-wing drones following slow-moving ground agents," Robotics and $\mathrm{Au}$ tonomous Systems, vol. 72, no. 1, pp. 285-294, 2015.

[23] N. Regina and M. Zanzi, "UAV guidance law for groundbased target trajectory tracking and loitering," in Proceedings of the 2011 IEEE Aerospace Conference, Big Sky, MT, USA, March 2011.

[24] H. K. Khalil, Nonlinear Systemspp. 139-144, Prentice Hall, Upper Saddle River, NJ, USA, 3rd edition, 2002. 